\title{
Scenarios of Higgs bosons and Z' manifestations in the minimal gauge extension of the SM
}

\section{Beylin*}

Research Institute of Physics, Southern Federal University, Russia

E-mail: vitbeylin@gmail.com

\section{A. Beylin}

Research Institute of Physics, Southern Federal University, Russia

E-mail: anbeylin@gmail.com

\section{A. Pivovarov}

Research Institute of Physics, Southern Federal University, Russia

E-mail: tex_k@mail.ru

\begin{abstract}
Two-gluon production of $t \bar{t} Z$ states at the LHC is analyzed with an account of intermediate Z' boson and two Higgs scalars. It is shown that interference effects in the distributions on the invariant mass of the $t \bar{t}$ pair are important. A large-scale interference picture is produced, in the fixed-width approach its details strongly depend on the model parameter. Distributions in $E_{b}$ for the decay $H \rightarrow t b W$ in the MSSM and in the minimal gauge extension of the SM are also compared for low and high $\tan \beta$.
\end{abstract}

The XXth International Workshop High Energy Physics and Quantum Field Theory September 24-October 1, 2011

Sochi, Russia

\footnotetext{
* Speaker.
} 


\section{Introduction}

There are specific features in the manifestations of the extended scalar sector which contains more than one Higgs boson. Self-coupling [1] and/or interference effects in a various channels naturally occur in the $2 \mathrm{HDM}$ model $[2,3]$, MSSM $[4,5]$ or in the minimal gauge extension of the SM based on the $S U(3) \times S U(2) \times U(1) \times U_{B-L}(1)$ group [6].

Indeed, to observe an extra Higgs bosons effects an analysis of differential distributions on the invariant mass, for example, is suitable. Namely, specific "peak-and-dip" structure arises near the Higgs boson mass as the result of interference of zero-order QCD diagrams and loops contributions in the gluon-gluon production of $t \bar{t}$ pairs. In the process, only Higgs boson intermediate state with a relatively small width $[7,8]$ was taken into account (interference effects of this type were firstly considered in $[9,10])$.

Interference terms can sufficiently change total cross section of the process considered namely, $\gamma-Z$ - interferencies and contributions increase annihilation cross section up to $10 \%$. At the same time, interference terms appear in the differential distributions as an extra peaks or peak-and dip structures near poles of intermediate states. Importantly, a complex and cumbersome interference picture is simplified if some interactions can be "switched off", as it takes place, for example, in the MSSM- $t \bar{t} H$ coupling is weakened for $\operatorname{tg} \beta>>1$. The interference structure is especially complex if there are some intermediate states which are close in mass. To consider role of interferencies in the distributions, the gauge SM extension with additional $U_{B-L}(1)$ group is useful and interesting due to small number of parameters in comparison with the MSSM, in particular. Moreover, an interesting peculiar regime of "strong coupling" emerges in the model leading to the fixed ratio of the Higgs boson masses.

\section{Some details on the $U_{B-L}(1)$ gauge extension of the SM}

The above mentioned minimal gauge extension of the SM contains one additional singlet Higgs boson, extra vector Z'-boson and three right-handed heavy neutrino [11-14], so it allows to consider neutrino oscillations as well. Two remained scalar states are mixed, consequently their mass spectrum depends on the scalar potential parameters $\lambda_{i}$ and on the mixing angle.

Potential energy of the scalar sector has the form

$$
V\left(H_{1}, H_{2}\right)=m_{1}^{2} H_{1}^{\dagger} H_{1}++m_{2}^{2}\left|H_{2}\right|^{2}+\lambda_{1}\left(H_{1}^{\dagger} H_{1}\right)^{2}+\lambda_{2}\left|H_{2}\right|^{4}+\lambda_{3} H_{1}^{\dagger} H_{1}\left|H_{2}\right|^{2} .
$$

From diagonalization of the mass form, the scalar bosons have following masses depending on the $\lambda_{i} v_{1,2}$ :

$$
\begin{gathered}
M_{H_{1}}^{2}=\lambda_{1} v_{1}^{2}+\lambda_{2} v_{2}^{2}-\left(\left(\lambda_{1} v_{1}^{2}-\lambda_{2} v_{2}^{2}\right)^{2}+\left(\lambda_{3} v_{1} v_{2}\right)^{2}\right)^{1 / 2} \\
M_{H_{2}}^{2}=\lambda_{1} v_{1}^{2}+\lambda_{2} v_{2}^{2}+\left(\left(\lambda_{1} v_{1}^{2}-\lambda_{2} v_{2}^{2}\right)^{2}+\left(\lambda_{3} v_{1} v_{2}\right)^{2}\right)^{1 / 2}
\end{gathered}
$$

These states of Higgs bosons are mixed, and the mixing angle is defined as

$$
\sin 2 \alpha=\frac{\lambda_{3} v_{1} v_{2}}{\left(\left(\lambda_{1} v_{1}^{2}-\lambda_{2} v_{2}^{2}\right)^{2}+\left(\lambda_{3} v_{1} v_{2}\right)^{2}\right)^{1 / 2}} .
$$



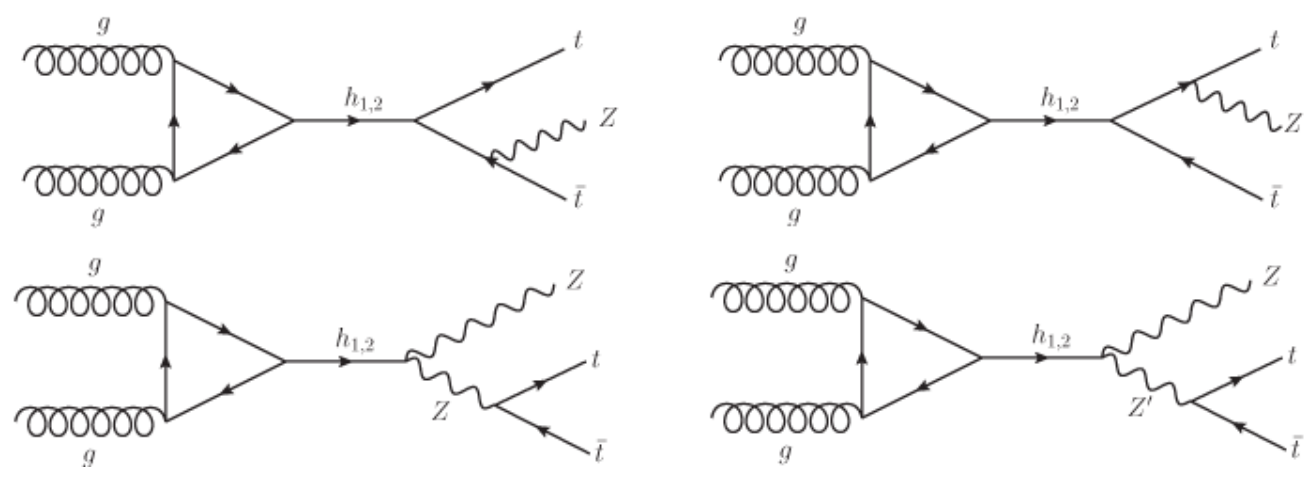

Figure 1: Contributions into $g g \rightarrow t \bar{t} Z$ reaction with two intermediate Higgs bosons

Note, specific "strong coupling regime" is realized in the Higgs sector when $\alpha \approx \Pi / 4$, i.e.

$$
\lambda_{1} v_{1}^{2} \approx \lambda_{2} v_{2}^{2} \approx \lambda_{3} v_{1} v_{2}
$$

It leads to the simple relation for the Higgs boson masses with an accuracy to loop corrections:

$$
M_{H_{1}}^{2} \approx 3 \cdot M_{H_{2}}^{2} .
$$

To study processes with $t \bar{t}, H_{i}, Z, Z^{\prime}$, we need in the following part of the model Lagrangian (all terms which are proportional to the small mixing angle, $\sim \sin \psi$, for $Z$ and $Z^{\prime}$ are omitted):

$$
\begin{array}{r}
L_{H}=-\frac{1}{2} g_{4}^{\prime}\left(g_{1}^{2}+g_{2}^{2}\right)^{1 / 2} Z_{\mu} Z^{\prime \mu} \cos \psi v_{1}\left(H_{1} \cos \alpha-H_{2} \sin \alpha\right) \\
+\frac{1}{4}\left(g_{1}^{2}+g_{2}^{2}\right) Z_{\mu} Z^{\mu} \cos \psi v_{1}\left(H_{1} \cos \alpha-H_{2} \sin \alpha\right)-\left(m_{t} t \bar{t}\right) \frac{1}{v_{1}}\left(H_{1} \cos \alpha-H_{2} \sin \alpha\right) .
\end{array}
$$

Using the Lagrangian, it is possible to analyze interference effects in the model with these two neutral Higgs bosons in various regimes and compare some processes in this SM modification with the MSSM. Rememeber, we use here the fixed-width approximation and omit K-factor, so the total error is estimated as $\sim 30 \%$. It does not change any qualitative conclusions on the interference structure manifestations and behaviour. To simplify the calculations, we use an analytic form of gluon distribution functions $g(x)=\frac{1}{n_{g}} x^{-a}(1-x)^{b}$ with coefficients depending on $Q^{2}$ in the form close to HERA approximation (see [15] and references therein).

\section{Interference effects in $p p \rightarrow t \bar{t} Z$}

The process under consideration is described in the SM by three diagrams mediated by the one Higgs boson intermediate state. However, in the models with the extended scalar sector and extra vector states there are eight diagrams with intermediate scalars and a lot of interference contributions, consequently. Here we limit ourselves by intermedite scalars only and omit possible s-channel $\gamma-Z-Z^{\prime}$ contributions and boxes.

As it was noted in $[7,8]$, if the width of the intermediate higgses is sufficiently large $\left(\sim\left(10^{1}-\right.\right.$ $\left.10^{2}\right) \mathrm{GeV}$ ) the narrow "peak-and-dip" near the s-channel intermediate mass should be smeared. For 

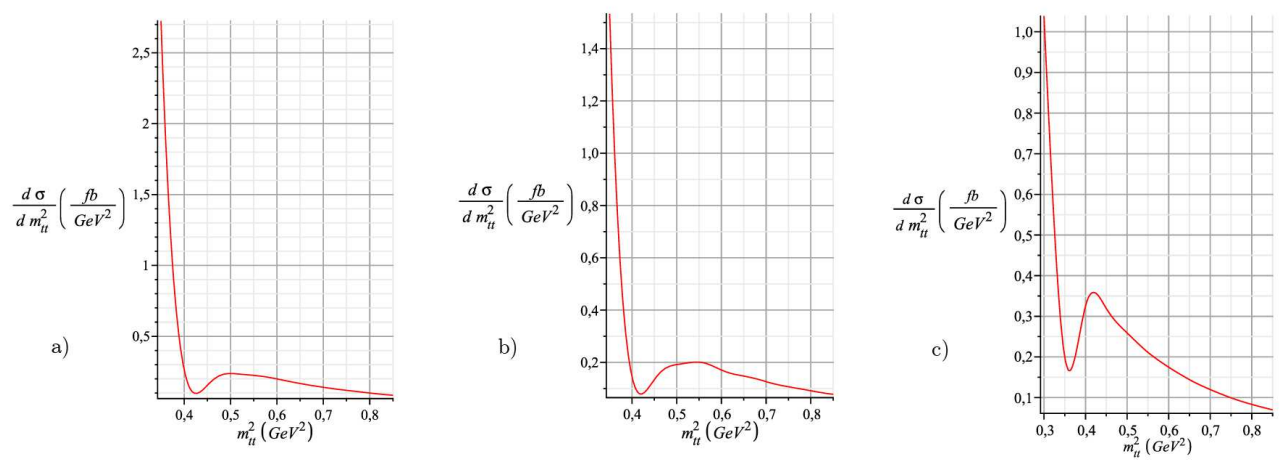

Figure 2: Distributions on $m_{t \bar{t}}^{2}$ for $M_{Z^{\prime}}=700 \mathrm{GeV}$ and a) $M_{H_{1}}=140 \mathrm{GeV}, M_{H_{2}}=600 \mathrm{GeV}, \alpha=\Pi / 3$; b) the same masses of higgses and $\alpha=\Pi / 6, \mathrm{c}) M_{H_{1}}=460 \mathrm{GeV}, M_{H_{2}}=820 \mathrm{GeV}, \alpha=\Pi / 3$

the case with numerous interference terms, especially if there are some states with close masses, the situation even worse. Then, the narrow "peak-and-dip" structure near the pole practically absent transforming into a broad interference picture. Some peaks induced there by (constructive and/or destructive) interference terms are joined into a large-scale structure in the invariant mass distribution. Note, however, the only energy region near the Z'-mass is presented here in the $t \bar{t}$ mass distribution. It means, we analyze a possibility to see some effects of $Z^{\prime}$ in the $t \bar{t} l l$ final states exploring $g g$-fusion channel with intermediate Higgs bosons. Of cource, $Z$ ' signal should be much more clean in the its s-channel production by $q \bar{q}$ or $g g$ with quark loops, however, the process allows to see what a complex picture of interferencies can emerge if all intermediate states are taken into account.

The structure is sensitive to the model parameters, i.e. there is an obvious dependence of the distribution on the type of the gauge SM extension — different masses of two Higgs bosons, mixing parameter values shift the interference peaks and change their amplitudes. Two possible values of Z' mass are used here: $700 \mathrm{GeV}$ and $3.5 \mathrm{TeV}$ (this value is preferred by $\mathrm{RG}$ analysis, see report of D. Vlasenko in this Workshop).

For the $M_{Z^{\prime}}=700 \mathrm{GeV}$ results of calcultions are presented in Fig.2(a,b,c); in all cases $Z^{\prime}$ coupling is defined in agreement with the experimental restriction $M_{Z^{\prime}} / g^{\prime} \geq 7 \mathrm{TeV}$; for the Higgs boson masses we take values that agree with the latest exclusion plot (august, 2011). More exactly, the recent data do not exclude (or, even, prefer) the Higgs boson mass lower then $\approx 140 \mathrm{GeV}$ (remind also, the lightest neutral Higgs boson in the MSSM should have $M_{h_{0}} \lesssim 130 \mathrm{GeV}$ ), at the same time the higgs mass should be larger than $\approx 470 \mathrm{GeV}$. So, in the model we can use both of the limits in a correspondence with different values of the mixing angle and parameters $\lambda_{1,2,3}$.

It is seen, near $M_{Z^{\prime}}^{2}$ value there is a broad peak which occurs due to contributions from numerous diagrams. Parameters of the model affect the peak width and height: they depend on the $\alpha$ for the case with one light and one heavy scalar state. But when both of Higgs bosons are heavy and their masses are not very far from $M_{Z^{\prime}}$ (in the "strong coupling regime"), the peak is more sharp, however its width remain large. Thus, in the distribution the large-scale interference structure is formed in the Z' mass vicinity with the smeared maximum; the structure details depend on the parameters obviously.

For the value $M_{Z^{\prime}}=3.5 \mathrm{TeV}$ and corresponding coupling $g_{\max }^{\prime}=0.5$ the $t \bar{t}$ mass distribution 

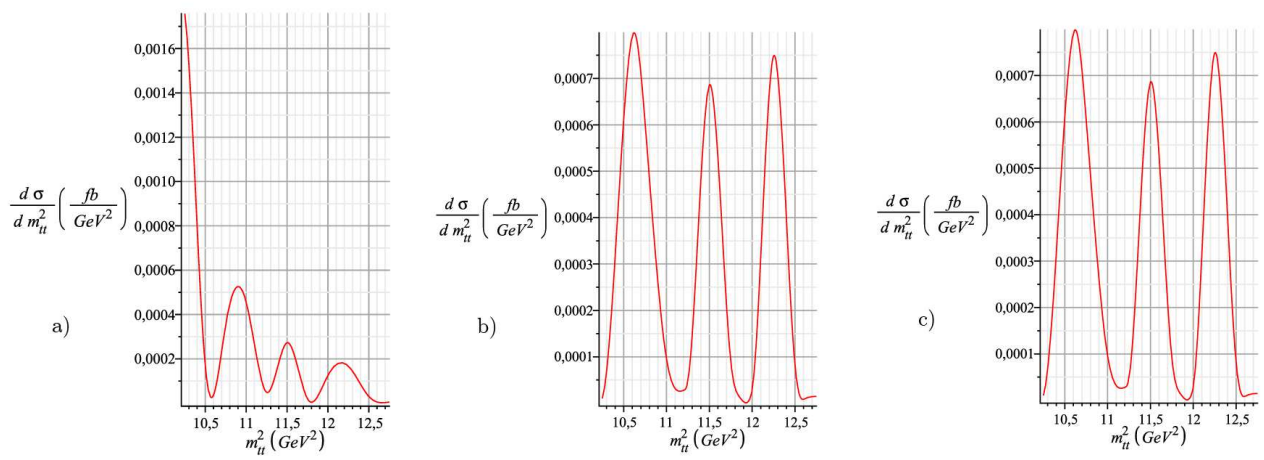

Figure 3: Distributions on $m_{t \bar{t}}^{2}$ for $M_{Z^{\prime}}=3.5 \mathrm{TeV}$ and a) $M_{H_{1}}=480 \mathrm{GeV}, M_{H_{2}}=820 \mathrm{GeV}, \alpha=\Pi / 6$; b) the same masses of higgses and $\alpha=\Pi / 4$, c) $M_{H_{1}}=140 \mathrm{GeV}, M_{H_{2}}=600 \mathrm{GeV}, \alpha=\Pi / 4$

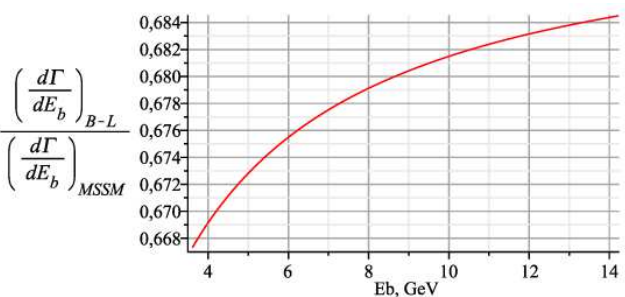

a)

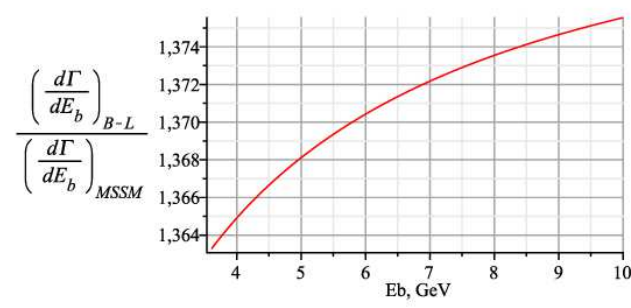

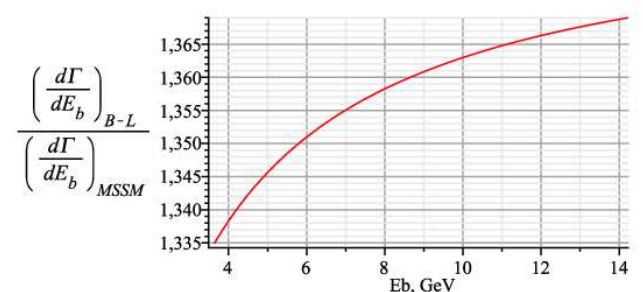

b)

Figure 4: Ratios of differential decays of Higgs boson in the MSSM with $\tan \beta=1.5$ and $U_{B-L}(1)$; a) $M_{H}=270 \mathrm{GeV}, \alpha=\Pi / 6$, b) $M_{H}=270 \mathrm{GeV}, \alpha=\Pi / 3, M_{H}=460 \mathrm{GeV}, \alpha=\Pi / 4$.

also demonstrates a set of interference peaks near $M_{Z^{\prime}}^{2}$ in the region $\sim \Gamma_{\text {tot }}\left(Z^{\prime}\right)$. These peaks are wide due to large $\Gamma_{t o t}\left(Z^{\prime}\right)$ and the center of the group of peaks shifts depending on the parameters - masses of scalars and $\alpha$. Remind, the calculations were done for the fixed widths of $H_{1,2}$ and $Z, Z^{\prime}$ bosons.

A comparison of differential in $E_{b}$ branchings for the decays $H \rightarrow t b W$ in the MSSM [16] and in the $U_{B-L}(1) \mathrm{SM}$ extension (Fig.4) demonstrates the ratio can be done $\sim 1$ for some value of model prameters and small $\tan \beta=1.5$. So, in some channels the data do not allow to distinguish the models with two neutral higgses. However, for $\tan \beta=30$ the ratio of branchings is very large and it remains nearly constant in this energy region (Fig.5). So, for large $\tan \beta$ these models, containing two neutral Higgs bosons, can be discriminated easily.

\section{Conclusions}

It has been shown, Z'-boson manifests in the model with two neutral Higgs bosons as an obvious signal near the resonance. The amplitude of the signal grows if the scalar masses are not 


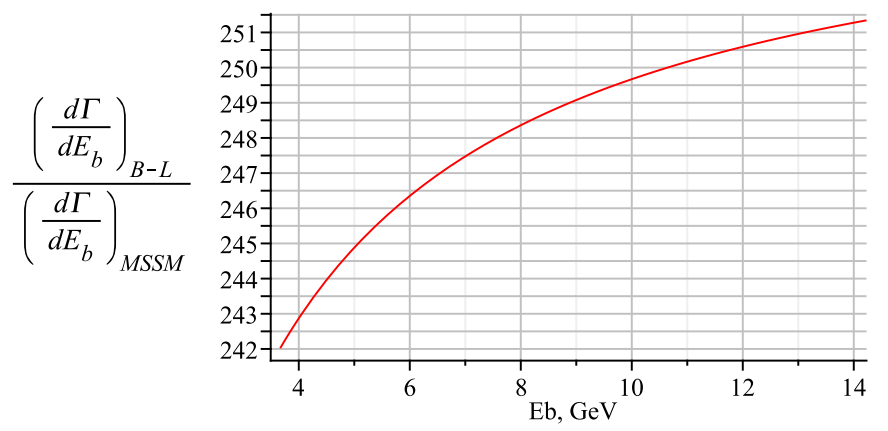

Figure 5: Ratio of differential decays of Higgs boson in the MSSM with $\tan \beta=30$ and $U_{B-L}(1) ; M_{H}=$ $270 \mathrm{GeV}, \alpha=\Pi / 4$.

very far from the Z' mass. However, Z' width is large, so the peak is very wide. Moreover, the peak is accompanied with an extra wide interference peaks resulting in some large-scale structure in the distribution in invariant $t \bar{t}$ mass. The peaks positions depend on the model parameters - masses of scalars and $\alpha$ - and on the fixed widths of bosons. To observe the signal in this reaction the whole interference picture should be studied in the energy region. The picture structure can change depending on the model parameters - widths, masses, couplings, mixing angles and vacuum shifts define the peaks positions and amplitudes. An analogous complex picture should occur in any case when there are some interfering (destructive and/or constructive)conributions with different couplings and and unstable intermediate states. So, from the consideration of $t \bar{t} Z$ final states at the LHC it is possible to extract some information on the structure of the Higgs sector and Z'-boson appearance.

This comment on the role of the interferencies in the differential distributions should be checked in details taking into account s-channel diagrams and boxes. Moreover, it is important to consider the energy dependence of the running widths for bosons in the $t \bar{t}$ production and corresponding asymmetries. Indeed, the results do not give a precision test because loop corrections (K-factor) and more exact PDF's should be taken into account together with supplementary diagrams. However, a general result is unchanged: consideration of the total interference region can shed some light on the parameters role and affection for the models with several Higgs bosons, especially.

\section{References}

[1] U. Baur, T. Plehn, D. Rainwater, Determining the Higgs Boson Self Coupling at Hadron Colliders, Phys. Rev. D67 (2003) 033003.

[2] V.D. Barger, J.L. Hewett, R.J.N. Phillips, New constraints on the charged Higgs sector in two-Higgs-doublet model, Phys. Rev. D41 (1990) 344.

[3] A. Djouadi, The anatomy of electro-weak symmetry breaking: The Higgs boson in the Standard Model. Part 1, Phys. Repts. 457 (2008) 1.

[4] J. Gunion, S. Dawson, H. Haber, G. Kane, The Higgs hunter's guide, Upton NY, Brookhaven Nat. Lab. (1989). 
[5] A. Djouadi, The anatomy of electro-weak symmetry breaking: The Higgs boson in the MSSM. Part 2, Phys. Repts. 459 (2008) 1.

[6] S. Khalil, Low scale B-L extension of the Standard Model at the LHC, J. Phys. G35 (2008) 05501.

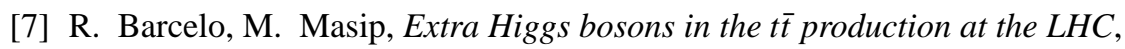
[hep-ph/1001.5456].

[8] D. Berdine, N. Kauer, D. Rainwater, Breakdown of the narrow-width approximation for new physics, Phys. Rev. Lett. 99 (2007) 111601.

[9] K.J.F. Gaemers, F. Hoogeven, Higgs production and decay into heavy flavours with the gluon fusion mechanism, Phys. Lett. B146 (1984) 347.

[10] D. Dicus, A. Stange, S. Willenbrock, Higgs decay to top quarks at hadron colliders, Phys. Lett. B333 (1994) 126.

[11] W. Emam, S. Khalil, Higgs and Z' phenomenology in B-L extension of the Standard Model at LHC, Eur. Phys. J. C52 (2007) 625.

[12] L. Basso, S. Moretti, G.M. Pruna, C.H. Shepherd-Themistocleous, Phenomenology of the minimal B-L extension of the Standard Model: Z' and neutrinos, Phys. Rev. D80 (2009) 055030.

[13] L. Basso, S. Moretti, G.M. Pruna, Phenomenology of the minimal B-L extension of the Standard Model: the Higgs sector, Phys. Rev. D83 (2011) 055014.

[14] L. Basso, A. Belyaev, S. Moretti, G.M. Pruna, C.H. Shepherd-Themistocleous, Z' discovery potential at the LHC in the minimal B-L extension of the Standard Model, Eur. Phys. J. C71 (2011) 1613.

[15] V. Aushev, Heavy flavours and QCD studies at HERA, in Proc. of QFTHEP-2011 (2011).

[16] A. Djouadi, J. Kalinowskiy, P.M. Zerwas, Two and Three Body Decay Modes of SUSY Higgs Particles, Z.Phys. C70 (1996) 435. 